\title{
Dynamics of quark-gluon plasma produced in heavy ion collisions
}

\author{
M. Ruggieri ${ }^{1, a}$, F. Scardina ${ }^{1,2}$, S. Plumari ${ }^{1,2}$, and V. Greco ${ }^{1,2}$ \\ ${ }^{1}$ Department of Physics and Astronomy, University of Catania, Via S. Sofia 64, I-95125 Catania \\ ${ }^{2}$ INFN-Laboratori Nazionali del Sud, Via S. Sofia 62, I-95123 Catania, Italy
}

\begin{abstract}
In this talk, we report on our results about the computation of isotropization and thermalization times of the quark-gluon plasma produced in relativistic heavy ion collisions, as well as of the elliptic flow. Simulation of the evolving fireball is achieved by solving the relativistic Boltzmann equation for the parton distribution function tuned at a fixed shear viscosity to entropy density ratio $\eta / s$. Our results show that the saturation in the initial spectrum reduces the efficiency in building-up the elliptic flow. Moreover both thermalization and isotropization times are quite small, approximately of $1 \mathrm{fm} / \mathrm{c}$, if the system is in a strong coupling regime.
\end{abstract}

\section{Introduction}

In the last decade it has been reached a general consensus that Ultra-relativistic heavy-ion collisions (uRHICs) at the Relativistic Heavy-Ion Collider (RHIC) and the Large Hadron Collider (LHC) create a hot and dense strongly interacting quark and gluon plasma (QGP) [1-4]. The QGP has a very small shear viscosity to entropy density, which is close to the lower bound of $1 / 4 \pi$ conjectured for systems at infinite strong coupling [5]. A key observable to reach such a conclusion is the elliptic flow [6], $v_{2}=\left\langle\left(p_{x}^{2}-p_{y}^{2}\right) /\left(p_{x}^{2}+p_{y}^{2}\right)\right\rangle$. The origin of $v_{2}$ is the initial spatial eccentricity of the overlap region in non-central collisions, which causes different pressure gradients in the transverse plane favouring flow preferably along one direction. The measured large $v_{2}$ is considered a signal of a very small $\eta / s$ because a large $\eta / s$ would damp flow, hence reducing anisotropy in momentum space. Calculations agree in indicating an average $\eta / s$ of the QGP lying in the range $4 \pi \eta / s \approx 1-3$ [7-19].

In this talk, we show our results on the computation of some properties of the QGP produced by uRHICs, performing in this sense an anatomy of the created fireball. In particular we report on temperature and pressures evolution, studying isotropization and thermalization times, as well as on differential elliptic flow, of the QGP fireball produced in relativistic heavy ion collisions. The evolution of the fireball is simulated by solving the relativistic Boltzmann equation for the parton distribution function tuned at a fixed shear viscosity to entropy density ratio $\eta / s[15,16,18,20]$. We find fast thermalization and isotropization times both at RHIC and LHC energies, both times being of the order of $1 \mathrm{fm} / \mathrm{c}$ or even smaller. However, even in presence of a fast thermalization, the initial out-of-equilibrium distribution proper of the KLN model has an effect on the final $v_{2}$, the effect being more important for peripheral collisions.

\footnotetext{
ae-mail: marco.ruggieri@lns.infn.it
} 


\section{Initial conditions for the simulations}

The results shown in this talk refer to three different initial conditions $[17,18]$. The first one is based on the thermal Glauber model, with a $\boldsymbol{p}$-space thermalized spectrum in the transverse plane at a time $\tau_{0}=0.6 \mathrm{fm} / \mathrm{c}$ for the RHIC runs and $\tau_{0}=0.3 \mathrm{fm} / \mathrm{c}$ for the LHC runs. We assume boost invariance in the longitudinal direction at $\tau=\tau_{0}$ which implies independence of the initial distribution on $y$. We will refer to this case as Th-Glauber.

The other two kinds of initial conditions we use in our simulations $[17,18]$ are based on a model of gluon production for the shattered Color Glass Condensate (CGC) [21] named factorized-KLN (fKLN in the following) [22-24], in which gluon distribution functions embed saturation effect at a scale $Q_{s}$ in the sense that they are constant for $p_{T}<Q_{s}$ and power law decreasing for $p_{T}>Q_{s}$. The second kind of initialization is obtained by distributing both in $p_{T}$-spectrum and the coordinate space by the fKLN model; we call this initialization as fKLN initialization. This kind of initial condition is not implemented in hydro simulations because the initial deviation from equilibrium cannot be studied within viscous hydrodynamics. In the fKLN initialization we assume $\tau_{0}=0.2 \mathrm{fm} / \mathrm{c}$ because no assumption on equilibration needs to be introduced; this time can be interpreted as the time needed for the strong initial fields to decay to a parton liquid. Finally we consider a third initialization which is close to the one usually implemented in hydro simulations, assuming the coordinate space distribution given by the KLN model and a $p_{T}$-thermalized spectrum like in the Glauber model. In this way we assume some thermalization occured in a short time, leaving at the same time the distribution in coordinate space unaffected. We call this initialization as Th-fKLN initialization. For the latter we assume the same initialization times used for the Th-Glauber model. For all cases the assumption $y=\eta$ is performed at $\tau=\tau_{0}$, with $\eta$ representing space-time rapidity.

\section{Kinetic theory at fixed $\eta / s$}

For the dynamical evolution of the initial conditions we employ transport theory as a base of a simulation code of the fireball expansion created in relativistic heavy-ion collision [15, 16, 20]; we assume the system can be described by a gluon distribution function $f(\boldsymbol{x}, \boldsymbol{p}, t)$ which evolves according to the Relativistic Boltzmann Transport (RBT) equation:

$$
p_{\mu} \partial^{\mu} f=\int d \Gamma_{2} d \Gamma_{1^{\prime}} d \Gamma_{2^{\prime}}\left(f_{1^{\prime}} f_{2^{\prime}}-f f_{2}\right)|\mathcal{M}|^{2} \delta^{4}\left(p+p_{2}-p_{1^{\prime}}-p_{2^{\prime}}\right),
$$

with $d^{3} \boldsymbol{p}_{k}=2 E_{k}(2 \pi)^{3} d \Gamma_{k}$, and $\mathcal{M}$ corresponds to the transition amplitude. At variance with the standard use of transport theory we have developed an approach that, instead of focusing on specific microscopic calculations of the scattering matrix, fixes the total cross section cell by cell in the grid in order to have the wished value of $\eta / s$. This approach offers a bridge between transport theory and hydrodynamics since we use the Boltzmann equation to simulate the evolution of a fluid which dissipates with a specified shear viscosity, in analogy to what is done within hydrodynamical simulations.

Using RBT equation the initial condition does not need to be close to thermal equilibrium, because it does not rely on a gradient expansion of the energy-momentum tensor. A similar approach has been also studied in [25] where it has been shown that transport theory at fixed $\eta / s$ reproduces the results of viscous hydrodynamics for one-body observable like $T^{\mu v}$ or entropy density also in the limit in which the system is not in the dilute regime. Despite the fact we consider only the $2 \leftrightarrow 2$ processes to compute the collision integral, once the system is close to the hydro regime with many collisions happening in a short time range, the detail of the single scattering is lost and what matters is only the viscosity of the fluid and not the specific microscopic process producing such viscosity, see also $[26,27]$. 

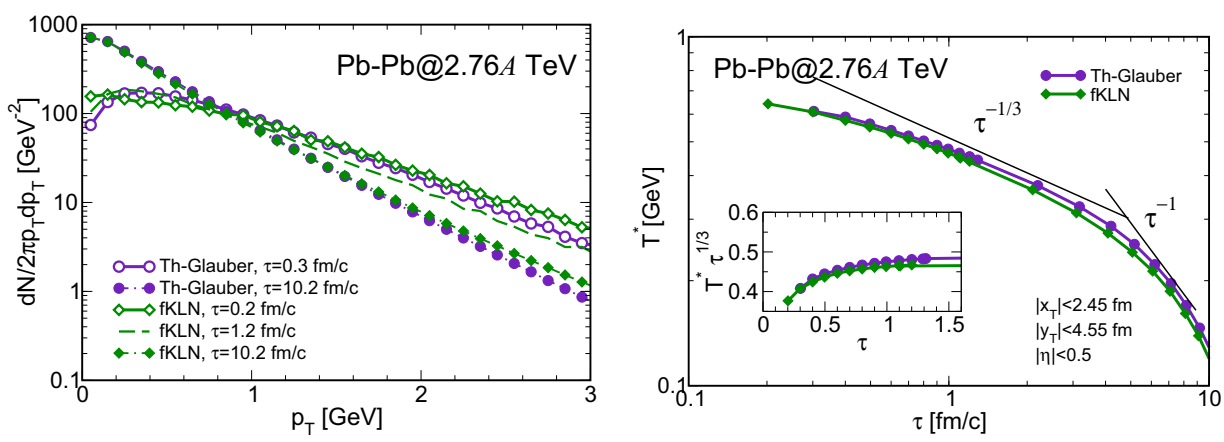

Figure 1. Left panel: Time evolution of spectra. Right panel: Time evolution of temperature. The spectra for the Th-fKLN case are not shown because we do not find visible deviations from the Th-Glauber initialization. Adapted from Ref. [18].

Once $\eta / s$ is fixed, we compute the total cross section in each cell of the coordinate space of our grid. To this end we need a relation between $\eta$, temperature, cross section and density; as shown in [20, 28], the Chapmann-Enskog approximation supplies such a relation with quite good approximation, in agreement with the results obtained using the Green Kubo formula. Therefore, we fix $\eta / s$ in each cell of the grid and compute the pertinent total cross section by means of the Chapmann-Enskog relation.

The $\eta / s$ we use in our study is as follows: in the plasma phase it is a constant, whose numerical value is fixed case by case; we then implement a kinetic freezout by assuming that $\eta / s$ increases smoothly in a temperature range from the plasma phase to a hadron phase values which is fixed by referring to the estimates in [29-31]. In this way we take into account scatterings in the hadron phase as well, which however give a very tiny contribution to the collective flow because of the damping due to the larger viscosity. For more details we refer to $[17,18]$.

\section{Thermalization and Isotropization}

In the left panel of Fig. 1 we collect the initial spectra for the case of a $\mathrm{Pb}-\mathrm{Pb}$ collision at $\sqrt{\mathrm{s}}=2.76 \mathrm{~A}$ $\mathrm{TeV}$ for the fKLN and Th-Glauber initial conditions at their respective initial times $\tau_{0}$ and at the final time $\tau=10.2 \mathrm{fm} / \mathrm{c}$. For the case of the Th-fKLN, we find that the spectra are the same as in the case of the Th-Glauber, therefore we do not plot them in the figure. We have also shown by the dashed line the spectrum at $\tau=1.2 \mathrm{fm} / \mathrm{c}$ for the case of the fKLN initial condition. The results in Fig. 1 are obtained with $4 \pi \eta / s=1$. The initial fKLN spectrum is not thermalized; nevertheless it evolves to a thermal one within $1 \mathrm{fm} / \mathrm{c}$, since its $p_{T}$ dependence becomes exponential with a slope very similar to the Th-Glauber. Thermalization is achieved in the full momentum space within $\tau_{\text {therm }} \approx 0.8 \mathrm{fm} / \mathrm{c}$; we check this by studying the time evolution of the quantity $T^{\star}=E / 3 N$ representing the temperature in the case of a thermalized system. We plot our result for $T^{\star}$ for the case of $\mathrm{Pb}-\mathrm{Pb}$ collisions at LHC energy in the right panel of Fig. 1. In fact in the case of 1D dissipation-less expansion of a thermalized medium $T^{\star} \propto \tau^{-1 / 3}$, which we find to happen for the fKLN case within $\tau_{\text {therm }}$, see the inset in the right panel of Fig. 1. For collisions at RHIC energy we find similar results.

In Fig. 2 we plot the ratio $P_{L} / P_{T}$ where $P_{L}, P_{T}$ correspond to the longitudinal and transverse pressure respectively. In each cell the pressures are computed in the local rest frame of the fluid, hence removing the pure flow contribution to the energy-momentum tensor. Results for both RHIC and LHC energies are shown. In the initial stage of the collision there is a strong anisotropy; however 


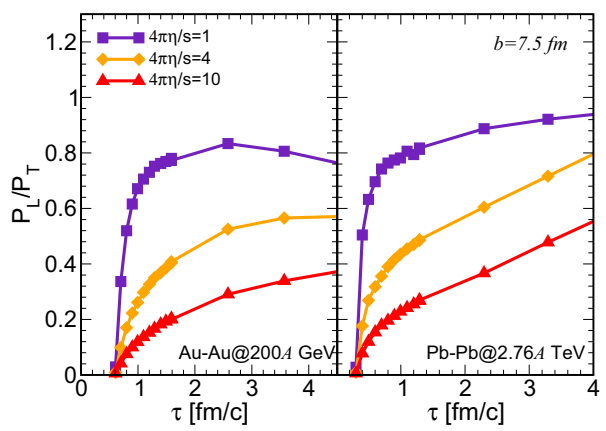

Figure 2. Time evolution of $P_{L} / P_{T}$ for the Th-Glauber initializations and several values of the ratio $\eta / s$, for RHIC and LHC energies. Adapted from Ref. [18].
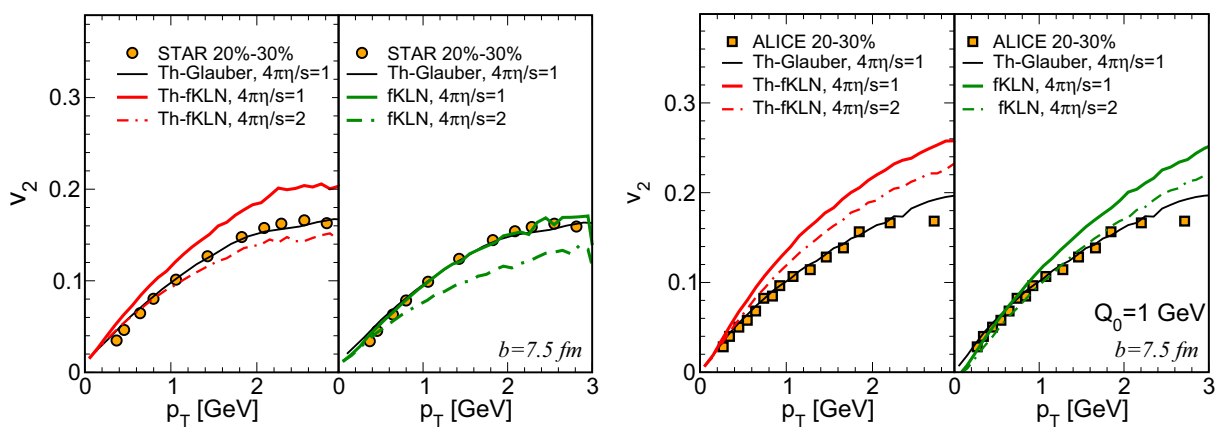

Figure 3. Elliptic flow $v_{2}\left(p_{T}\right)$ at midrapidity $|y|<0.5$ for different initial conditions and $\eta / s$ as in the legend. Adapted from Ref. [18].

the system efficiently removes the anisotropy in the case of small $\eta / s$, and in fact we find that the time required to get isotropic pressure $P_{L} \approx P_{T}$ is $\tau_{\text {iso }} \approx 0.3 \mathrm{fm} / \mathrm{c}$. On the other hand in case $\eta / s$ is quite large and comparable with the perturbative QCD estimates, the system is not efficient in removing the initial anisotropy, which is quite natural because the large viscosity damps the flow which instead is necessary to transfer momentum and equilibrate the pressures.

\section{Elliptic flow}

In the left panel of Fig. 3, we collect our results for the differential elliptic flow for the case of a Au-Au collision at RHIC energy with $b=7.5 \mathrm{fm}$; in the center panel we plot the same quantity for the case of $\mathrm{Pb}-\mathrm{Pb}$ collisions at LHC energy and same impact parameter. Since no hadronization process is yet included in our approach, the comparison with the experimental charged hadrons $v_{2}[32,33]$ has to be taken with care, even if it indicates that the azimuthal asymmetries generated by RBT are in the correct range.

For the cases of Th-Glauber and Th-fKLN initializations at RHIC energy the $v_{2}$ obtained by ThGlauber with $4 \pi \eta / s=1$ is obtained by Th-fKLN with $4 \pi \eta / s \approx 2$, because momentum distribution is the same but initial eccentricity of Th-fKLN is larger than the one of the Th-Glauber initial condition, 


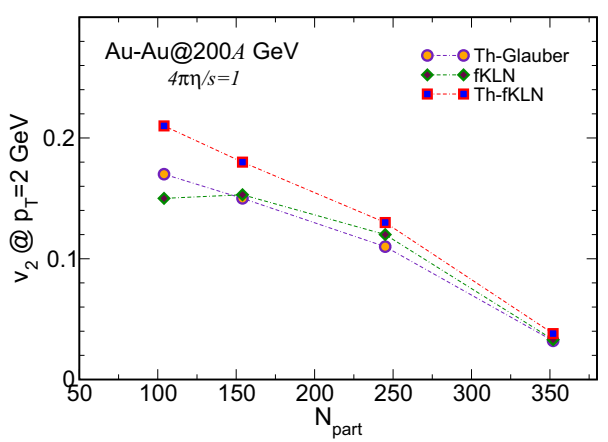

Figure 4. Elliptic flow $v_{2}\left(p_{T}\right)$ at midrapidity $|y|<0.5$ for different initial conditions and $\eta / s$ as in the legend and for $p_{T}=2 \mathrm{GeV}$. Adapted from Ref. [18].

requiring a larger viscosity in the former case to damp the flow. These results are in agreement with the ones obtained from viscous hydrodynamics $[9,34,35]$. In the case of the fKLN initialization, represented by the green lines in Fig. 3, we find that fKLN with a $4 \pi \eta / s=1$ gives a $v_{2}\left(p_{T}\right)$ quite similar to the Th-Glauber, even if the initial eccentricity in this case is larger. Hence the initial out-ofequilibrium fKLN distribution reduces the efficiency in converting the initial spatial eccentricity into $v_{2}$. We obtain similar results at the LHC energy, see center panel of Fig. 3.

The effect we find on $v_{2}$ is more important for peripheral collisions. In fact in Fig. 4 we plot $v_{2}$ at $p_{T}=2 \mathrm{GeV}$ for different initializations, as a function of the number of participants. This figure permits to summarize the dependence of $v_{2}\left(p_{T}\right)$ on the centrality class, comparing the impact of the initial distribution on the final $v_{2}$. The discrepancy between Th-fKLN and fKLN initializations becomes less relevant for more central collisions, implying that the effect of the initial momentum distribution is not negligible if one considers non-central collisions.

\section{Conclusions}

In this talk we have reported our results on thermalization, isotropization and building-up of the elliptic flow for QGP produced in relativistic heavy ion collisions both at RHIC and LHC energies. Our study is based on kinetic theory at fixed $\eta / s$. Fixing $\eta / s$ permits to forget about microscopic processes which give that specific value of the ratio itself, and to focus on the effect of shear viscosity on thermalization, isotropization and building of the collective flows.

In both RHIC and LHC runs we have found that thermalization and isotropization times are both of the order of $1 \mathrm{fm} / \mathrm{c}$ or even smaller in the case of strong coupling ( $\operatorname{small} \eta / s$ ); in the opposite case of weak coupling the system is not able to remove initial anisotropy. For what concerns the elliptic flow we have found that it depends not only on the initial pressure gradients and the $\eta / s$ of the system, but also on the initial distribution in momentum space. In particular, an initial condition characterized by a momentum distribution with a saturation scale generates smaller $v_{2}$ respect to the thermal one. We find that this effect is maximal for semi-peripheral collisions, becoming quite small for very central collisions. 


\section{References}

[1] STAR, J. Adams et al., Nucl. Phys. A757, 102 (2005); PHENIX, K. Adcox et al., Nucl. Phys. A757, 184 (2005).

[2] K. Aamodt et al. [ALICE Collaboration], Phys. Rev. Lett. 105, 252302 (2010).

[3] B. V. Jacak and B. Muller, Science 337, 310 (2012).

[4] R. J. Fries, V. Greco and P. Sorensen, Ann. Rev. Nucl. Part. Sci. 58 (2008) 177

[5] P. Kovtun, D. T. Son, and A. O. Starinets, Phys. Rev. Lett. 94, 111601 (2005).

[6] J. Y. Ollitrault, Phys. Rev. D 46, 229 (1992).

[7] P. Romatschke and U. Romatschke, Phys. Rev. Lett. 99, 172301 (2007).

[8] H. Song and U.W. Heinz, Phys. Rev. C 78, 024902 (2008).

[9] H. Song, S. A. Bass, U. Heinz, T. Hirano and C. Shen, Phys. Rev. C 83, 054910 (2011).

[10] B. Schenke, S. Jeon and C. Gale, Phys. Rev. C 82 (2010) 014903

[11] H. Niemi, G. S. Denicol, P. Huovinen, E. Molnar and D. H. Rischke, Phys. Rev. Lett. 106 (2011) 212302

[12] Z. Xu and C. Greiner, Phys. Rev. C 79 (2009) 014904

[13] Z. Xu, C. Greiner and H. Stocker, Phys. Rev. Lett. 101 (2008) 082302

[14] E. L. Bratkovskaya, W. Cassing, V. P. Konchakovski and O. Linnyk, Nucl. Phys. A 856 (2011) 162

[15] G. Ferini, M. Colonna, M. Di Toro and V. Greco, Phys. Lett. B 670, 325 (2009)

[16] S. Plumari and V. Greco, AIP Conf. Proc. 1422 (2012) 56

[17] M. Ruggieri, F. Scardina, S. Plumari and V. Greco, Phys. Lett. B 727, 177 (2013).

[18] M. Ruggieri, F. Scardina, S. Plumari and V. Greco, Phys. Rev. C 89, 054914 (2014).

[19] S. K. Das and J. e. Alam, Phys. Rev. D 83, 114011 (2011) [arXiv:1011.4181 [nucl-th]].

[20] S. Plumari, A. Puglisi, M. Colonna, F. Scardina and V. Greco, J. Phys. Conf. Ser. 420, 012029 (2013).

[21] L. D. McLerran and R. Venugopalan, Phys. Rev. D 49, 2233 (1994); Phys. Rev. D 49, 3352 (1994); Phys. Rev. D 50, 2225 (1994).

[22] D. Kharzeev, E. Levin and M. Nardi, Nucl. Phys. A 747, 609 (2005); D. Kharzeev and M. Nardi, Phys. Lett. B 507, 121 (2001); D. Kharzeev and E. Levin, Phys. Lett. B 523, 79 (2001); T. Hirano and Y. Nara, Nucl. Phys. A 743, 305 (2004).

[23] H. -J. Drescher and Y. Nara, Phys. Rev. C 75, 034905 (2007); Phys. Rev. C 76, 041903 (2007).

[24] T. Hirano and Y. Nara, Phys. Rev. C 79, 064904 (2009)

[25] P. Huovinen and D. Molnar, Phys. Rev. C 79, 014906 (2009).

[26] V. Greco, Anisotropic Flow from a Kinetic Theory Ap- proach, Talk given at INPC 2013, 2-6 June 2013, Florence (Italy).

[27] S. Plumari, A. Puglisi, F. Scardina and V. Greco, J. Phys. Conf. Ser. 446, 012025 (2013).

[28] S. Plumari, A. Puglisi, F. Scardina and V. Greco, Phys. Rev. C 86 (2012) 054902.

[29] M. Prakash, M. Prakash, R. Venugopalan, and G. Welke, Phys. Rept. 227, 321 (1993).

[30] J.-W. Chen, Y.-H. Li, Y.-F. Liu, and E. Nakano, Phys. Rev. D76, 114011 (2007).

[31] N. Demir and S. A. Bass, Phys.Rev.Lett. 102, 172302 (2009).

[32] J. Adams et al. [STAR Collaboration], Phys. Rev. C 72, 014904 (2005).

[33] A. Dobrin, J. Phys. G 38, 124170 (2011).

[34] A. Adare et al. [PHENIX Collaboration], Phys. Rev. Lett. 107, 252301 (2011).

[35] M. Luzum and P. Romatschke, Phys. Rev. C 78, 034915 (2008). 